\title{
A New Approach to Model Updating for Symmetric Structures
}

\author{
Jonathan L. du Bois* and Nick A. J. Lieven ${ }^{\dagger}$ \\ University of Bristol, Queens Building, University Walk, Bristol, BS8 1TR, England \\ and Sondipon Adhikari ${ }^{\ddagger}$ \\ Swansea University, Singleton Park, Swansea SA2 8PP, United Kingdom
}

In this paper a new technique is proposed for updating parameters in a Finite Element (FE) model based upon modal coupling. Modal coupling exists between vibration modes with respect to parametric variations and is often associated with curve veering and vibration localisation.

Examination of the modal coupling leads to the development of a new set of system properties, providing a different emphasis for updating schemes. In particular, the properties convey information about the system which cannot be deduced from the eigenvalues alone, yet can be measured experimentally more accurately than the eigenvectors. This information is found to be especially useful in nominally symmetric or periodic systems where parameters may be indeterminable using traditional techniques. It is thought that the new methods may find particular application in the tuning of bladed rotor assemblies.

The presentation begins with a discussion of veering and localisation, and its manifestation in symmetric structures. The modal coupling and related properties are then derived, in tandem with methods of extracting these quantities from experimental data. Next, the FE model and updating scheme are outlined, and to conclude an experimental example is given, demonstrating the arrival at a unique solution to an otherwise ambiguous updating problem.

\section{Nomenclature}

Symbols

$\beta \quad$ angle between current eigenvector set and datum eigenvector set in the mass-normalised basis

$\delta_{j} \quad$ arbitrary system parameter

$\kappa_{i j k} \quad$ modal coupling for $i^{t h}$ and $k^{t h}$ eigenvalues with respect to parameter $\delta_{j}$

$\lambda_{i} \quad i^{\text {th }}$ eigenvalue

$\mu_{\bar{\lambda}} \quad$ mean eigenvalue at the veering datum

$\sigma_{i j i} \quad$ sensitivity of $i^{\text {th }}$ eigenvalue to parameter $\delta_{j}$

$\Delta \delta_{j} \quad$ discrepancy between current parameter value and datum parameter value, $\bar{\delta}_{j}-\delta_{j}$

$\Delta \lambda_{k i} \quad$ separation of $k^{t h}$ and $i^{t h}$ eigenvalues, $\lambda_{k}-\lambda_{i}$

$\Delta \sigma_{k j i} \quad$ difference in sensitivities of modes $k$ and $i$ to parameter $\delta_{j}, \sigma_{k j k}-\sigma_{i j i}$

$\boldsymbol{\Lambda}_{i k} \quad$ diagonal matrix of $i^{t h}$ and $k^{t h}$ eigenvalues

$\boldsymbol{\Phi}_{i k} \quad$ matrix of $i^{t h}$ and $k^{t h}$ mass-normalised eigenvectors

$\boldsymbol{\Sigma}_{i j k} \quad$ sensitivity matrix for $i^{t h}$ and $k^{t h}$ modes

p quadratic curve fit parameters describing veering characteristics

$\mathbf{q} \quad$ linear curve fit parameters describing mean eigenvalue locus

K $\quad$ stiffness matrix

M mass matrix

${ }^{*}$ PhD Student, Department of Aerospace Engineering, University of Bristol, Student Member Grade.

$\dagger$ Dean, Engineering Faculty, University of Bristol

${ }^{\ddagger}$ Chair of Aerospace Engineering, School of Engineering, Swansea University 
veering characteristic sensitivity matrix used in updating scheme rotational transform matrix

overbar denotes properties at the veering datum

\section{Introduction}

Localisation $^{1}$ and eigenvalue curve veering ${ }^{2}$ are two phenomena that are commonly observed under parametric variation of a dynamic system, particularly in symmetric or periodic structures. ${ }^{3}$ Both behaviours are related to the interaction of the vibration modes within the metasystem formed by the inclusion of the parameter dynamics. As two eigenvalue loci converge they may veer suddenly away from one another, simultaneously swapping properties and then continuing on their exchanged trajectories. In these regions the mode shapes must transform rapidly but smoothly from one to another. This interaction is facilitated by modal coupling with respect to the parameter variation ${ }^{4}$ (unrelated to the coupling sometimes associated with non-proportional damping). Perturbation of symmetric or periodic structures will often induce such modal coupling, distorting the mode shapes and producing localised vibrations.

While the manifestation of these behaviours is often inconsequential, occuring only for very specific configurations, there are situations in which the ramifications are significant. Notably, localisation may lead to far greater vibration levels than predicted by idealised models, for example in turbine rotors. ${ }^{5}$ Similarly, the modal interactions may provide indicators of other behaviour such as stability. ${ }^{6}$ In the modelling of such systems it is important that the veering and localisation behaviour is represented accurately.

Finite element (FE) model updating, when applied intelligently, provides a means of adapting numerical models to account for unknown physical parameters. Sometimes these are introduced as a result of simplified models, where the behaviour of a complex substructure may be adequately represented by only a few elements. Alternatively, the parameters could be difficult to measure individually and thus better determined using the updating process. The measured data from dynamic testing is compared to the analytical results and the discrepancies are minimised to produce a more accurate numerical representation of the system.

Most commonly, the eigenvalues are used as the measured data in updating schemes. Dependent upon the nature and number of the parameters being updated, the eigenvalues alone may not be sufficient. This is particularly true for symmetric structures where many parameters may have identical influences on the eigenvalues, thus rendering the updating problem ill-conditioned. In this case the natural frequencies may be complemented by the mode shapes, although this data is generally considered to be less reliable.

This paper derives new quantities, directly related to the modal coupling, which may be used to update a model to produce an accurate representation of the veering behaviour. They take advantage of the accuracy of the measured eigenvalues and the qualitative insights offered by the eigenvectors. The application of these quantities in a sensitivity-based updating scheme is described, and a method for obtaining the values from experimental data is outlined before demonstrating the technique with respect to real experimental data.

\section{Background}

The analysis of the modal interactions herein is based upon the transformations of the eigenvectors throughout a veering region. Extending the observations of Balmès, ${ }^{7}$ a conference paper by the authors ${ }^{8}$ explains how a wide class of veering modes may be described in terms of eigenvector rotations within a fixed subspace. The pertinent findings are summarised below for linear stiffness variations, as will be observed in the later sections of this paper.

For proximate modes, the transformations of the eigenvectors can be expressed approximately as a simple rotation within a fixed subspace of the normal basis:

$$
\overline{\boldsymbol{\Phi}}_{i k}=\boldsymbol{\Phi}_{i k} \mathbf{T}, \quad \mathbf{T}=\left[\begin{array}{rr}
\cos \beta & -\sin \beta \\
\sin \beta & \cos \beta
\end{array}\right]
$$

where $\boldsymbol{\Phi}_{i k}$ is the mass normalised eigenvector matrix for the $i^{t h}$ and $k^{t h}$ modes. For perfectly isolated modes with no interaction with other modes, the vectors rotate asymptotically through $90^{\circ}$. The midway point in this rotation will be referred to as the veering datum, and it is at this point that the eigenvalue sensitivities are equal, the curvature is maximum and the rate of eigenvector rotation is highest. The vector set $\overline{\boldsymbol{\Phi}}_{i k}$ will be used to denote this datum set and the overbar will consistently denote properties at the datum. 
A sensitivity matrix for modes $i$ and $k$ can be defined as

$$
\boldsymbol{\Sigma}_{i j k}=\boldsymbol{\Phi}_{i k}^{T} \frac{d \mathbf{K}}{d \delta_{j}} \boldsymbol{\Phi}_{i k}-\boldsymbol{\Phi}_{i k}^{T} \frac{d \mathbf{M}}{d \delta_{j}} \boldsymbol{\Phi}_{i k} \boldsymbol{\Lambda}_{i k}=\left[\begin{array}{cc}
\sigma_{i j i} & \kappa_{i j k} \\
\kappa_{k j i} & \sigma_{k j k}
\end{array}\right]
$$

where $\mathbf{M}$ and $\mathbf{K}$ are the mass and stiffness matrices, $\boldsymbol{\Lambda}_{i k}$ is a diagonal matrix of the $i^{\text {th }}$ and $k^{t h}$ eigenvalues, the diagonal terms are the sensitivities of the eigenvalues to parameter $\delta_{j}$ and the off-diagonal terms are the modal coupling responsible for the veering behaviour. The variation of these properties with the vector rotations is described by

$$
\begin{aligned}
\sin (2 \beta) & =\Delta \sigma_{k j i} / 2 \bar{\kappa}_{i j k} \\
\cos (2 \beta) & =\kappa_{i j k} / \bar{\kappa}_{i j k} \\
\bar{\kappa}_{i j k}^{2} & =\kappa_{i j k}{ }^{2}+\left(\Delta \sigma_{k j i} / 2\right)^{2}
\end{aligned}
$$

where $\Delta \sigma_{k j i}=\sigma_{k j k}-\sigma_{i j i}$ and $\bar{\kappa}_{i j k}$ is the maximum, or datum, value for the modal coupling. The next sections build on this foundation to derive other characteristic quantities of the veering system.

\section{Veering Parameter Datum}

A subset of the equations of motion for an undamped dynamic system is given by

$$
\mathbf{K} \boldsymbol{\Phi}_{i k}-\mathbf{M} \boldsymbol{\Phi}_{i k} \boldsymbol{\Lambda}_{i k}=\mathbf{0}
$$

Considering a linear variation of the stiffness matrix, the datum configuration is expressed as $\overline{\mathbf{K}}=\mathbf{K}+$ $\frac{d \mathbf{K}}{d \delta_{K}} \Delta \delta_{K}$ where $\Delta \delta_{K}=\bar{\delta}_{K}-\delta_{K}$. The equation of motion for the datum system can then be written

$$
\left(\mathbf{K}+\frac{d \mathbf{K}}{d \delta_{K}} \Delta \delta_{K}\right) \overline{\boldsymbol{\Phi}}_{i k}-\overline{\mathbf{M \Phi}}_{i k} \overline{\mathbf{\Lambda}}_{i k}=\mathbf{0}
$$

A constant mass matrix is assumed so that the transformation of the mass-normalised eigenvectors takes the form of a rotation, $\beta$, in the orthonormal basis thus defined. Premultiplying by $\overline{\boldsymbol{\Phi}}_{i k}^{T}$ and using eqn. (1) gives

$$
\mathbf{T}^{T} \boldsymbol{\Phi}_{i k}^{T}\left(\mathbf{K}+\frac{d \mathbf{K}}{d \delta_{K}} \Delta \delta_{K}\right) \boldsymbol{\Phi}_{i k} \mathbf{T}-\overline{\boldsymbol{\Phi}}_{i k}^{T} \overline{\mathbf{M}}_{i k} \overline{\mathbf{\Lambda}}_{i k}=\mathbf{0}
$$

Applying orthogonal relationships to eqn. 8 and using eqn. (2) with $\frac{d \mathbf{M}}{d \delta_{K}}=0$, the modified eigenvalues can be expressed in terms of the initial modal properties so that

$$
\overline{\boldsymbol{\Lambda}}_{i k}=\mathbf{T}^{T} \boldsymbol{\Lambda}_{i k} \mathbf{T}+\mathbf{T}^{T} \boldsymbol{\Sigma}_{i K k} \mathbf{T} \Delta \delta_{K}
$$

where the diagonal elements of the matrix equation give

$$
\begin{aligned}
\bar{\lambda}_{i}= & \lambda_{i} \cos ^{2}(\beta)+\lambda_{k} \sin ^{2}(\beta) \\
& +\left(\sigma_{i K i} \cos ^{2}(\beta)+2 \kappa_{i K k} \cos (\beta) \sin (\beta)+\sigma_{k K k} \sin ^{2}(\beta)\right) \Delta \delta_{K} \\
\bar{\lambda}_{k}= & \lambda_{i} \sin ^{2}(\beta)+\lambda_{k} \cos ^{2}(\beta) \\
& +\left(\sigma_{i K i} \sin ^{2}(\beta)-2 \kappa_{i K k} \cos (\beta) \sin (\beta)+\sigma_{k K k} \cos ^{2}(\beta)\right) \Delta \delta_{K},
\end{aligned}
$$

and the off-diagonal elements identically prescribe

$$
\Delta \lambda_{k i} \cos (\beta) \sin (\beta)+\left(\Delta \sigma_{k K i} \cos (\beta) \sin (\beta)+\kappa_{i K k}\left(\cos ^{2}(\beta)-\sin ^{2}(\beta)\right)\right) \Delta \delta_{K}=0
$$

where $\Delta \lambda_{k i}=\lambda_{k}-\lambda_{i}$. Rearranging the latter and applying trigonometric identities allows the determination of the parameter variation required to produce a given vector rotation,

$$
\Delta \delta_{K}=-\frac{\Delta \lambda_{k i} \tan (2 \beta)}{\Delta \sigma_{k K i} \tan (2 \beta)+2 \kappa_{i K k}}
$$

and substitution of eqns. (3-5) gives the veering parameter datum as

$$
\bar{\delta}_{K}=\delta_{K}-\frac{\Delta \lambda_{k i} \Delta \sigma_{k K i}}{4 \bar{\kappa}_{i K k}^{2}}
$$




\section{Eigenvalues}

It is now possible to determine the eigenvalues at the veering datum. Rearranging eqns. (10) and (11) and applying trigonometric identities gives

$$
\begin{aligned}
\bar{\lambda}_{i}= & \frac{\lambda_{i}+\lambda_{k}}{2}-\frac{\Delta \lambda_{k i}}{2} \cos (2 \beta) \\
& +\left(\frac{\sigma_{i K i}+\sigma_{k K k}}{2}-\frac{\Delta \sigma_{k K i}}{2} \cos (2 \beta)+\kappa_{i K k} \sin (2 \beta)\right)\left(\bar{\delta}_{K}-\delta_{K}\right) \\
\bar{\lambda}_{k}= & \frac{\lambda_{i}+\lambda_{k}}{2}+\frac{\Delta \lambda_{k i}}{2} \cos (2 \beta) \\
& +\left(\frac{\sigma_{i K i}+\sigma_{k K k}}{2}+\frac{\Delta \sigma_{k K i}}{2} \cos (2 \beta)-\kappa_{i K k} \sin (2 \beta)\right)\left(\bar{\delta}_{K}-\delta_{K}\right) .
\end{aligned}
$$

Using eqns. (3-5),

$$
\begin{aligned}
& \bar{\lambda}_{i}=\frac{\lambda_{i}+\lambda_{k}}{2}-\frac{\Delta \lambda_{k i}}{2} \frac{\kappa_{i K k}}{\bar{\kappa}_{i K k}}+\left(\frac{\sigma_{i K i}+\sigma_{k K k}}{2}\right)\left(\bar{\delta}_{K}-\delta_{K}\right) \\
& \bar{\lambda}_{k}=\frac{\lambda_{i}+\lambda_{k}}{2}+\frac{\Delta \lambda_{k i}}{2} \frac{\kappa_{i K k}}{\bar{\kappa}_{i K k}}+\left(\frac{\sigma_{i K i}+\sigma_{k K k}}{2}\right)\left(\bar{\delta}_{K}-\delta_{K}\right) .
\end{aligned}
$$

Substituting eqn. (14),

$$
\begin{aligned}
& \bar{\lambda}_{i}=\frac{\lambda_{i}+\lambda_{k}}{2}-\frac{\left(\sigma_{i K i}+\sigma_{k K k}\right) \Delta \lambda_{k i} \Delta \sigma_{k K i}}{8 \bar{\kappa}_{i K k}^{2}}-\frac{\Delta \lambda_{k i} \kappa_{i K k}}{2 \bar{\kappa}_{i K k}} \\
& \bar{\lambda}_{k}=\frac{\lambda_{i}+\lambda_{k}}{2}-\frac{\left(\sigma_{i K i}+\sigma_{k K k}\right) \Delta \lambda_{k i} \Delta \sigma_{k K i}}{8 \bar{\kappa}_{i K k}^{2}}+\frac{\Delta \lambda_{k i} \kappa_{i K k}}{2 \bar{\kappa}_{i K k}} .
\end{aligned}
$$

A useful quantity is the separation of the eigenvalues at their closest point, the veering datum. From the difference of eqns. (20) and (21),

$$
\frac{\Delta \lambda_{k i}}{\Delta \bar{\lambda}_{k i}}=\frac{\bar{\kappa}_{i K k}}{\kappa_{i K k}}
$$

This simple result provides a powerful means of determining the minimum eigenvalue separation of two veering modes.

Finally, the mean eigenvalue at the veeering datum is easily found from eqns. (20) and (21) as

$$
\mu_{\bar{\lambda}}=\frac{\bar{\lambda}_{i}+\bar{\lambda}_{k}}{2}=\frac{\lambda_{i}+\lambda_{k}}{2}-\frac{\left(\sigma_{i K i}+\sigma_{k K k}\right) \Delta \lambda_{k i} \Delta \sigma_{k K i}}{8 \bar{\kappa}_{i K k}^{2}} .
$$

\section{Vector Rotation Rate}

The aim of this study is to determine updating variables which succinctly characterise the behaviour of two veering modes. The mean eigenvalue, parameter value and eigenvalue separation at the veering datum are all useful quantities but do not convey all of the necessary information.

The maximum modal coupling, $\bar{\kappa}_{i K k}$, seems an obvious choice for describing the coupling between the modes. Significantly, it conveys the direction of rotation of the eigenvectors, which is critical in differentiating between symmetric parameters. In practice, however, the modal coupling at the peak of veering will often show little variation over a wide range of coupling configurations. From eqns. (3-5), in the limit, $\beta \rightarrow 45^{\circ}$ and $\kappa_{i K k} \rightarrow 0$ so $\bar{\kappa}_{i K k} \rightarrow \Delta \sigma_{k K i}$. Thus, the maximum modal coupling is simply a measure of the difference in gradients of the two asymptotic eigenvalue loci and does not provide any real measure of the actual coupling between the modes.

Instead, the parameter chosen to represent the coupling is the rate of eigenvector rotation within the subspace, $\mathrm{d} \beta / \mathrm{d} \delta_{K}$. It is related to, but not entirely equivalent to, the eigenvector sensitivities. Importantly, 
the maximum rotation rate, $\overline{\mathrm{d} \beta / \mathrm{d} \delta_{K}}$, occurs at the veering datum and conveys both the magnitude and sign of the coupling. Rearranging eqn. (14) and differentiating yields

$$
\frac{\mathrm{d} \delta_{K}}{\mathrm{~d} \beta}=\frac{\Delta \sigma_{k i}}{4 \bar{\kappa}_{i K k}^{2}} \frac{\mathrm{d} \Delta \lambda_{k i}}{\mathrm{~d} \beta}+\frac{\Delta \lambda_{k i}}{4 \bar{\kappa}_{i K k}^{2}} \frac{\mathrm{d} \Delta \sigma_{k i}}{\mathrm{~d} \beta} .
$$

Eqns. (3), (4) and (22) produce

$$
\Delta \sigma_{k i}=2 \bar{\kappa}_{i K k} \sin (2 \beta) \quad \text { and } \quad \Delta \lambda_{k i}=\frac{\Delta \bar{\lambda}_{k i}}{\cos (2 \beta)}
$$

so that differentiating gives

$$
\frac{\mathrm{d} \Delta \sigma_{k i}}{\mathrm{~d} \beta}=4 \bar{\kappa}_{i K k} \cos (2 \beta) \quad \text { and } \quad \frac{\mathrm{d} \Delta \lambda_{k i}}{\mathrm{~d} \beta}=2 \frac{\Delta \bar{\lambda}_{k i}}{\cos ^{2}(2 \beta)} \sin (2 \beta) .
$$

Substituting eqns. (3), (4) and (22) again,

$$
\frac{\mathrm{d} \Delta \sigma_{k i}}{\mathrm{~d} \beta}=4 \kappa_{i K k} \quad \text { and } \quad \frac{\mathrm{d} \Delta \lambda_{k i}}{\mathrm{~d} \beta}=\frac{\Delta \sigma_{k i} \Delta \lambda_{k i}}{\kappa_{i K k}} .
$$

Combining eqns. (27) and (24) and rearranging yields

$$
\frac{\mathrm{d} \delta_{K}}{\mathrm{~d} \beta}=\frac{\Delta \lambda_{k i}}{\bar{\kappa}_{i K k}^{2}}\left(\frac{\left(\Delta \sigma_{k i} / 2\right)^{2}+\kappa_{i K k}^{2}}{\kappa_{i K k}}\right),
$$

and using eqn. (5) this becomes

$$
\frac{\mathrm{d} \delta_{K}}{\mathrm{~d} \beta}=\frac{\Delta \lambda_{k i}}{\kappa_{i K k}} .
$$

The eigenvector rotation rate is then given by

$$
\frac{\mathrm{d} \beta}{\mathrm{d} \delta_{K}}=\left(\frac{\mathrm{d} \delta_{K}}{\mathrm{~d} \beta}\right)^{-1}=\frac{\kappa_{i K k}}{\Delta \lambda_{k i}}
$$

and the maximum rotation rate by

$$
\frac{\overline{\mathrm{d} \beta}}{\mathrm{d} \delta_{K}}=\frac{\bar{\kappa}_{i K k}}{\Delta \bar{\lambda}_{k i}} .
$$

Although this function is the most physically recognisable, it is discontinuous across $\Delta \bar{\lambda}_{k i}=0$, making its

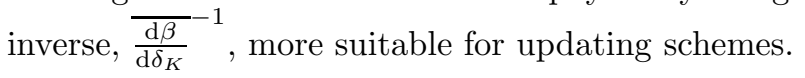

\section{Updating Scheme}

A sensitivity-based updating scheme is to be employed for this study, as described by Friswell and Mottershead. ${ }^{9}$ The premise is to gather experimental data from a series of tests, varying an independent parameter such that the results capture the modal properties throughout a veering region. The veering characteristics described above may then be calculated (as detailed in the next section), and one or more updating parameters (separate from the independent parameter) may be determined. In order to accomplish this task, the sensitivities of the veering characteristics must be determined with respect to the updating parameters.

The first step is to obtain the derivative of the eigenvalue sensitivities and modal coupling, contained within the sensitivity matrix. Differentiating eqn. (2) with respect to an arbitrary parameter $\delta_{p}$ and remembering $\frac{d \mathrm{M}}{d \delta_{K}}=0$ produces

$$
\frac{d \boldsymbol{\Sigma}_{i K k}}{d \delta_{p}}=\left[\begin{array}{cc}
\frac{d \sigma_{i K i}}{d \delta_{p}} & \frac{d \kappa_{i K k}}{d \delta_{p}} \\
\frac{d \kappa_{k K i}}{d \delta_{p}} & \frac{d \sigma_{k K k}}{d \delta_{p}}
\end{array}\right]=\frac{d \boldsymbol{\Phi}_{i k}^{T}}{d \delta_{p}} \frac{d \mathbf{K}}{d \delta_{K}} \boldsymbol{\Phi}_{i k}+\boldsymbol{\Phi}_{i k}^{T} \frac{d \mathbf{K}}{d \delta_{K}} \frac{d \boldsymbol{\Phi}_{i k}}{d \delta_{p}},
$$


where the parameters $\delta_{K}$ and $\delta_{p}$ are assumed to be independent so that $\frac{d^{2} \mathbf{K}}{d \delta_{K} d \delta_{p}}=0$. The eigenvalue derivative $\frac{d \boldsymbol{\Phi}_{i k}}{d \delta_{p}}$ is best obtained with Nelson's method. ${ }^{10}$ The eigenvalue derivatives are obtained with Fox and Kapoor's equation, ${ }^{11}$ and the following relationships are noted:

$$
\begin{aligned}
& \frac{d \Delta \lambda_{k i}}{d \delta_{p}}=\frac{d \lambda_{k}}{d \delta_{p}}-\frac{d \lambda_{i}}{d \delta_{p}} \\
& \frac{d \Delta \sigma_{k i}}{d \delta_{p}}=\frac{d \sigma_{k}}{d \delta_{p}}-\frac{d \sigma_{i}}{d \delta_{p}} .
\end{aligned}
$$

The sensitivity of the maximum modal coupling is determined by differentiating eqn. (5) to produce

$$
\frac{d}{d \delta_{p}} \bar{\kappa}_{i K k}^{2}=2 \kappa_{i K k} \frac{d \kappa_{i K k}}{d \delta_{p}}+\frac{\Delta \sigma_{k i}}{2} \frac{d \Delta \sigma_{k i}}{d \delta_{p}}
$$

and hence

$$
\frac{d}{d \delta_{p}} \bar{\kappa}_{i K k}=\frac{\kappa_{i K k}}{\bar{\kappa}_{i K k}} \frac{d \kappa_{i K k}}{d \delta_{p}}+\frac{\Delta \sigma_{k i}}{4 \bar{\kappa}_{i K k}} \frac{d \Delta \sigma_{k i}}{d \delta_{p}}
$$

The sensitivity of the minimum eigenvalue separation is found by differentiating eqn. (22), yielding

$$
\frac{d \Delta \bar{\lambda}_{k i}}{d \delta_{p}}=\frac{\kappa_{i K k}}{\bar{\kappa}_{i K k}} \frac{d \Delta \lambda_{k i}}{d \delta_{p}}+\frac{\Delta \lambda_{k i}}{\bar{\kappa}_{i K k}} \frac{d \kappa_{i K k}}{d \delta_{p}}-\frac{\Delta \lambda_{k i} \kappa_{i K k}}{\bar{\kappa}_{i K k}^{2}} \frac{d \bar{\kappa}_{i K k}}{d \delta_{p}} .
$$

It is now possible to determine the derivative of the maximum vector rotation rate, or more specifically its inverse, from eqn. (31):

$$
\frac{\partial}{\partial \delta_{p}}\left({\frac{d \beta^{-1}}{d \delta_{K}}}^{-1}\right)=\frac{1}{\bar{\kappa}_{i K k}} \frac{d \Delta \bar{\lambda}_{k i}}{d \delta_{p}}-\frac{\Delta \bar{\lambda}_{k i}}{\bar{\kappa}_{i K k}^{2}} \frac{d \bar{\kappa}_{i K k}}{d \delta_{p}} .
$$

Similarly the derivative of the datum parameter value is found by rearranging and differentiating eqn. (14) to give

$$
\frac{d \bar{\delta}_{K}}{d \delta_{p}}=\frac{\Delta \sigma_{k i} \Delta \lambda_{k i}}{4 \bar{\kappa}_{i K k}^{4}} \frac{\partial}{\partial \delta_{p}} \bar{\kappa}_{i K k}^{2}-\frac{\Delta \sigma_{k i}}{4 \bar{\kappa}_{i K k}^{2}} \frac{d \Delta \lambda_{k i}}{d \delta_{p}}-\frac{\Delta \lambda_{k i}}{4 \bar{\kappa}_{i K k}^{2}} \frac{d \Delta \sigma_{k i}}{d \delta_{p}} .
$$

Finally, eqn. (23) is differentiated to give

$$
\begin{aligned}
\frac{d \mu_{\bar{\lambda}}}{d \delta_{p}}= & \frac{1}{2}\left(\frac{d \lambda_{i}}{d \delta_{p}}+\frac{d \lambda_{k}}{d \delta_{p}}\right)-\frac{\Delta \lambda_{k i} \Delta \sigma_{k i}}{8 \bar{\kappa}_{i K k}^{2}}\left(\frac{d \sigma_{i}}{d \delta_{p}}+\frac{d \sigma_{k}}{d \delta_{p}}\right) \\
& -\frac{\left(\sigma_{i}+\sigma_{k}\right) \Delta \sigma_{k i}}{8 \bar{\kappa}_{i K k}^{2}} \frac{d \Delta \lambda_{k i}}{d \delta_{p}}-\frac{\left(\sigma_{i}+\sigma_{k}\right) \Delta \lambda_{k i}}{8 \bar{\kappa}_{i K k}^{2}} \frac{d \Delta \sigma_{k i}}{d \delta_{p}} \\
& +\frac{\left(\sigma_{i}+\sigma_{k}\right) \Delta \lambda_{k i} \Delta \sigma_{k i}}{8 \bar{\kappa}_{i K k}^{4}} \frac{d}{d \delta_{p}} \bar{\kappa}_{i K k}^{2} .
\end{aligned}
$$

Eqns. (14), (23), (31), (38), (39) and (40) may be combined to produce the necessary sensitivity calculations for the updating scheme. Importantly, these calculations may be performed using the modal results from a single eigensolution, performed anywhere in the region of the veering datum. The characteristics of the veering behaviour are captured in the single solution without the need for iterative solutions, making the scheme computationally efficient.

\section{Experimental Data Processing}

To implement this technique, experimental data must be obtained for a range of independently controlled parameter values spanning the veering region. Curve fitting methods are then used to extract the veering characteristics from the data. The curve fitting is performed using the eigenvalue separation, $\Delta \lambda_{k i}$, computed directly as the difference of the measured eigenvalues. From eqns. (5), (14) and (22),

$$
\Delta \lambda_{k i}^{2}=\left(4 \bar{\kappa}_{i K k}^{2}\right) \delta_{K}^{2}-\left(8 \bar{\kappa}_{i K k}^{2} \bar{\delta}_{K}\right) \delta_{K}+\left(4 \bar{\kappa}_{i K k}^{2} \bar{\delta}_{K}^{2}+\Delta \bar{\lambda}_{k i}^{2}\right)
$$


Thus in the vicinity of the veering, $\Delta \lambda_{k i}^{2}$ should be described by a quadratic in the controlled parameter $\delta_{K}$. The least squares curve fit is obtained from

$$
\mathbf{p}=\left[\begin{array}{ccc}
\delta_{K}^{(1)^{2}} & \delta_{K}^{(1)} & 1 \\
\delta_{K}^{(2)^{2}} & \delta_{K}^{(2)} & 1 \\
\vdots & \vdots & \vdots \\
\delta_{K}^{(n)^{2}} & \delta_{K}^{(n)} & 1
\end{array}\right]^{+}\left\{\begin{array}{c}
\Delta \lambda_{k i}^{(1)^{2}} \\
\Delta \lambda_{k i}^{(2)} \\
\vdots \\
\Delta \lambda_{k i}^{(n)^{2}}
\end{array}\right\}
$$

where bracketed superscripts denote the experimental data point, + denotes the pseudo-inverse such that

$$
\mathbf{A}^{+}=\left(\mathbf{A}^{T} \mathbf{A}\right)^{-1} \mathbf{A}^{T},
$$

and

$$
\mathbf{p}=\left\{\begin{array}{c}
4 \bar{\kappa}_{i K k}^{2} \\
-8 \bar{\kappa}_{i K k}^{2} \bar{\delta}_{K} \\
4 \bar{\kappa}_{i K k}^{2} \bar{\delta}_{K}^{2}+\Delta \bar{\lambda}_{k i}^{2}
\end{array}\right\} .
$$

From eqn. (44) the veering properties $\left|\bar{\kappa}_{i K k}\right|, \bar{\delta}_{K}$ and $\Delta \bar{\lambda}_{k i}$ may be established in turn, and all that remains is to determine the the sign of $\bar{\kappa}_{i K k}$. This is not possible from consideration of the eigenvalues alone, and the eigenvectors must be consulted.

There are several methods available for establishing the sign of $\bar{\kappa}_{i K k}$, the simplest being inspection of the eigenvector rotations. Having established a set of reference vectors, the eigenvector orientations can be determined using a dot product with each of the reference vectors. Their rotation should be plotted as a smooth curve, and from eqn. (30), positive modal coupling corresponds with positive $d \beta / d \delta_{K}$ (and hence negative vector rotation direction as $\beta$ is the angle from $\boldsymbol{\Phi}_{i k}$ to $\overline{\boldsymbol{\Phi}}_{i k}$ ). This distinction allows the determination of parameters which would otherwise be insoluble due to symmetry.

In order to describe the veering eigenvalue curves fully, two more properties are required; these are both provided by the mean eigenvalue locus, which traces a straight line under linear stiffness variation. The least squares solution is

$$
\mathbf{q}=\left[\begin{array}{cc}
\delta_{K}^{(1)} & 1 \\
\delta_{K}^{(2)} & 1 \\
\vdots & \vdots \\
\delta_{K}^{(n)} & 1
\end{array}\right]^{+}\left\{\begin{array}{c}
\mu_{\lambda}^{(1)} \\
\mu_{\lambda}^{(2)} \\
\vdots \\
\mu_{\lambda}^{(n)}
\end{array}\right\}
$$

where $\mu_{\lambda}^{(n)}=\left(\lambda_{i}^{(n)}+\lambda_{k}^{(n)}\right) / 2$. The most obvious properties, and those which are adopted here, are the gradient of this line and the mean eigenvalue at the veering datum, given by

$$
\frac{\mathrm{d} \mu_{\lambda}}{\mathrm{d} \delta_{K}}=q_{1} \quad \text { and } \quad \mu_{\bar{\lambda}}=\bar{\delta}_{K} q_{1}+q_{2} .
$$

\section{Welded Frame Example}

It is anticipated that model updates based on the veering properties will produce the best results when these properties are used in conjunction with more commonplace updating variables, notably the eigenvalues. For this example, however, the veering properties will be used in isolation to demonstrate their utility. To this end, an experimental rig in the form of a cross-braced rectangle is employed. The structure is seen in Fig. 1. Two bolts in one of the cross-members allow tensioning of the structure which induces stress-stiffening, a geometric nonlinearity whereby the transverse stiffness of slender elements is affected by axial loading. A detailed description of the experimental setup and results can be found in previous publications. ${ }^{2,12}$ The critical finding is that several eigenvalue loci intersect as the loading is varied, and those of modes 5 and 6 are found to veer around $3500 \mathrm{~N}$. This is in contrast to the symmetric analytical model where the loci cross. Differences in the weld stiffnesses joining the members at the corners are the most likely cause for discrepancies and this example will seek to determine these parameters. 


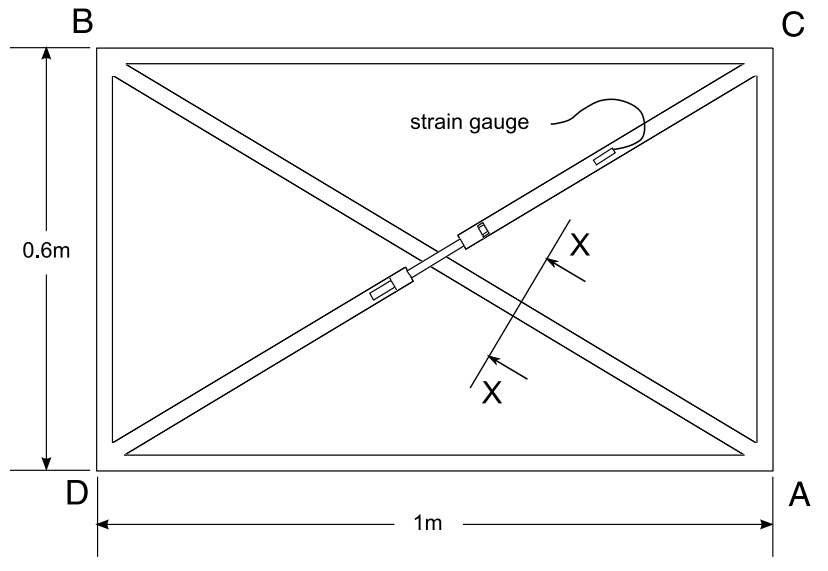

(a) Top view

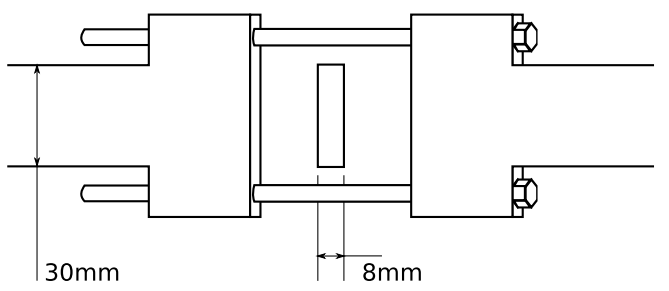

(b) Section X-X

Figure 1. Cross-braced rectangular frame used for updating tests.

To perform the updating computations as detailed above, the tangent stiffness derivative $\frac{d \mathbf{K}}{d \delta_{K}}$ must be known. Again, full details of the numerical model may be found in reference, ${ }^{12}$ and a numerical finite difference technique is now used to obtain the tangent stiffness derivative:

$$
\frac{d \mathbf{K}}{d \delta_{K}}=\frac{\mathbf{K}^{(n+1)}-\mathbf{K}^{(n)}}{\delta_{K}^{(n+1)}-\delta_{K}^{(n)}}
$$

where the bracketed superscipts refer to the load step.

The squared eigenvalue separation is plotted in fig. 2. The quadratic trend is clear, but the mild nonlinearity of the structural loading causes a shift over the course of the loading range. For this reason the data points used in the curve fitting should be limited to the vicinity of the veering. In contrast, it is desirable to include sufficient points so as to produce a reliable estimate of the true curve.

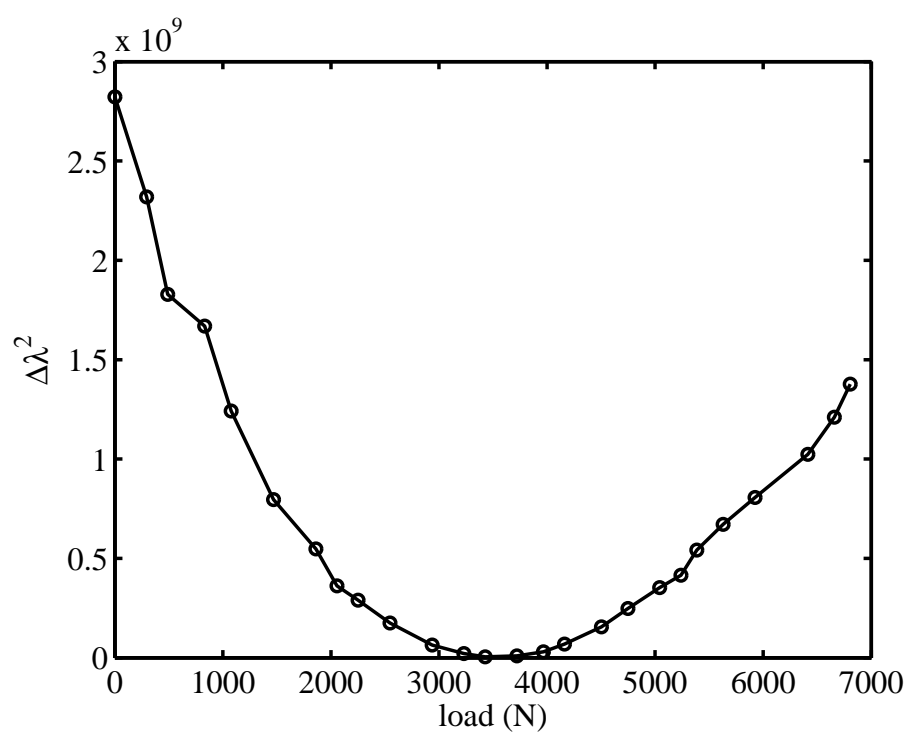

Figure 2. Experimentally determined eigenvalue separation of modes 5 and 6 in the welded frame.

Fig. 3 shows the variation of the modal properties as the load range is expanded to encompass more data points. It is found that between the $1000 \mathrm{~N}$ and $3000 \mathrm{~N}$ ranges the estimates settle to reasonable values before diverging to the invalid global least squares fit. Accordingly modal property values are chosen based on the estimates from this range, as indicated in fig. 3. These values produce the quadratic curve in 
fig. 4, corresponding with the eigenvalue loci in fig. 5. They exhibit a good approximation, with significant differences emerging only at the outermost extents of the loading spectrum.

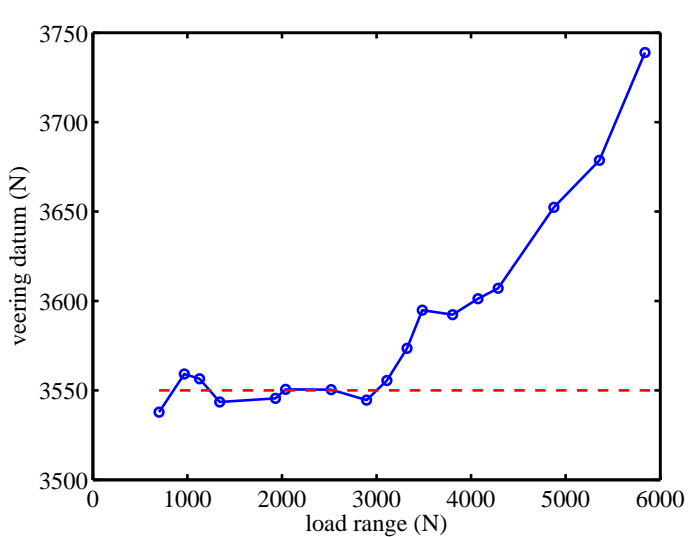

(a) Veering Parameter Datum

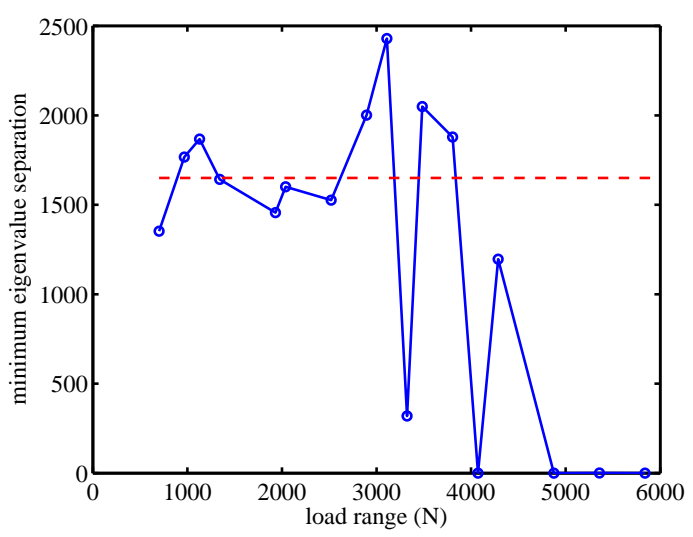

(c) Minimum Eigenvalue Separation

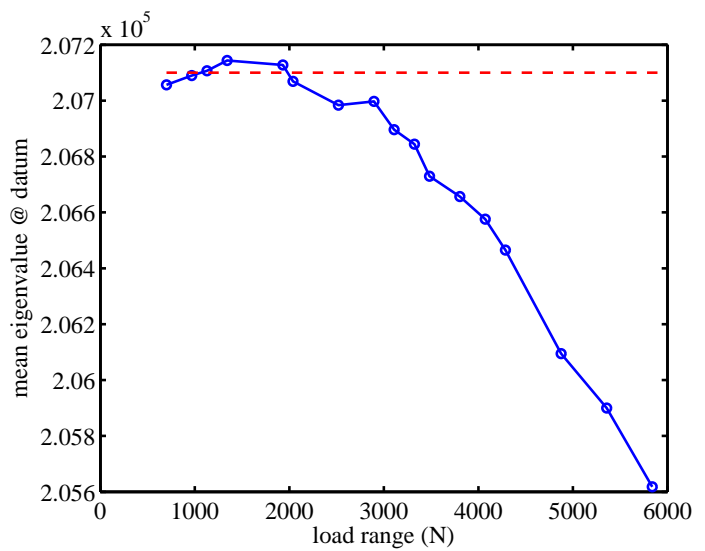

(b) Mean Eigenvalue at Veering Parameter Datum

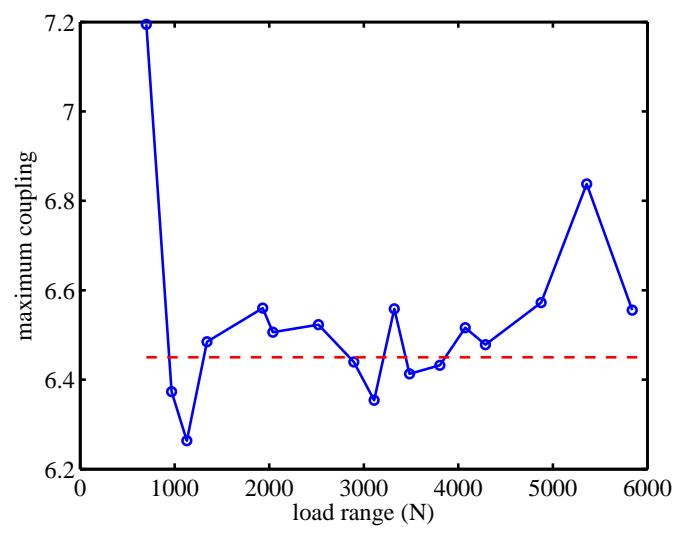

(d) Maximum Modal Coupling

Figure 3. Veering properties extracted from the experimental data using data points from varying load ranges, centered approximately about the veering datum. The values chosen for the updates are indicated by the dashed lines.

In the FE model, the weld stiffnesses connecting the diagonal members to the rest of the frame are parameterised using the cross-sectional width of short beam elements at the ends of each member. Using traditional eigenvalue-based techniques the symmetry of the structure prevents the determination of a unique solution to the update for more than one corner. Inclusion of the vector rotation rate resolves this difficulty, as the sign of the rotation rate differentiates between the effects of symmetrical parameters. Despite the additional information given by the vector rotation rate, the structure is almost symmetrical about two axes, so it is still not posssible to update all four parameters simultaneously. This information deficiency would likely be resolved by incorporting the veering properties for two further modes, but this example will simply consider the two parameter pairs separately. It has been recognised that the welds do not form perfectly rigid joints, so the four parameters are initialised to $70 \%$ of the full stiffness.

To update two parameters at least two measured quantities must be used. The first of these will be the maximum vector rotation rate. For the second, two quantities will be considered here in turn: the veering parameter datum and the mean eigenvalue at veering. The sensitivity matrix to be used in the update is then

$$
\mathbf{S}=\left[\begin{array}{cccc}
\frac{\partial}{\partial \delta_{A}}\left({\overline{\frac{d \beta}{d \delta_{K}}}}^{-1}\right) & \frac{\partial}{\partial \delta_{B}}\left({\overline{\frac{d \beta}{d \delta_{K}}}}^{-1}\right) & \frac{\partial}{\partial \delta_{C}}\left({\overline{\frac{d \beta}{d \delta_{K}}}}^{-1}\right) & \frac{\partial}{\partial \delta_{D}}\left({\overline{\frac{d \beta}{d \delta_{K}}}}^{-1}\right) \\
\frac{\partial \bar{\delta}_{K}}{\partial \delta_{A}} & \frac{\partial \bar{\delta}_{K}}{\partial \delta_{B}} & \frac{\partial \bar{\delta}_{K}}{\partial \delta_{C}} & \frac{\partial \bar{\delta}_{K}}{\partial \delta_{D}} \\
\frac{\partial \mu_{\lambda}}{\partial \delta_{A}} & \frac{\partial \mu_{\lambda}}{\partial \delta_{B}} & \frac{\partial \mu_{\lambda}}{\partial \delta_{C}} & \frac{\partial \mu_{\lambda}}{\partial \delta_{D}}
\end{array}\right]
$$

where the subscripts A-D refer to the welds at locations A-D in fig. 1(a), and where only two columns and 


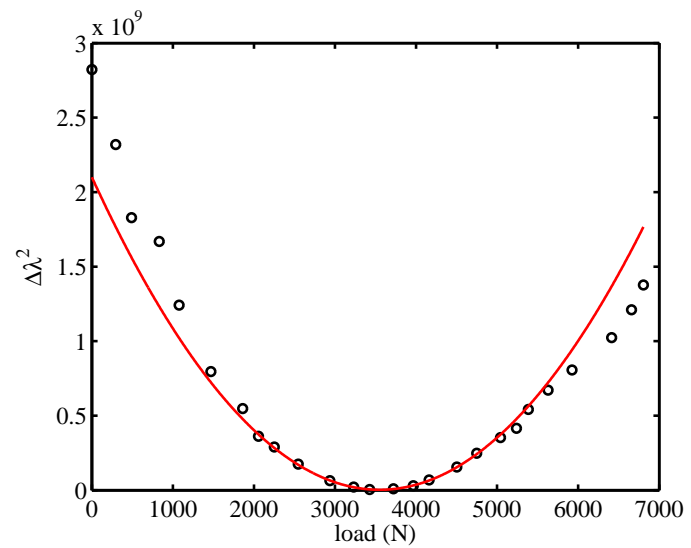

(a) Full range

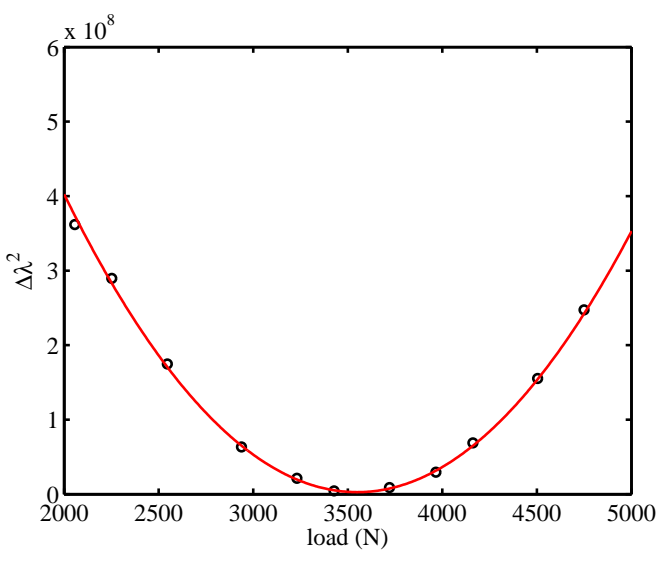

(b) Veering Region

Figure 4. The quadratic curve produced with the chosen veering property values. The fit is very good in the veering region, at the expense of the fit at the extents of the loading range.

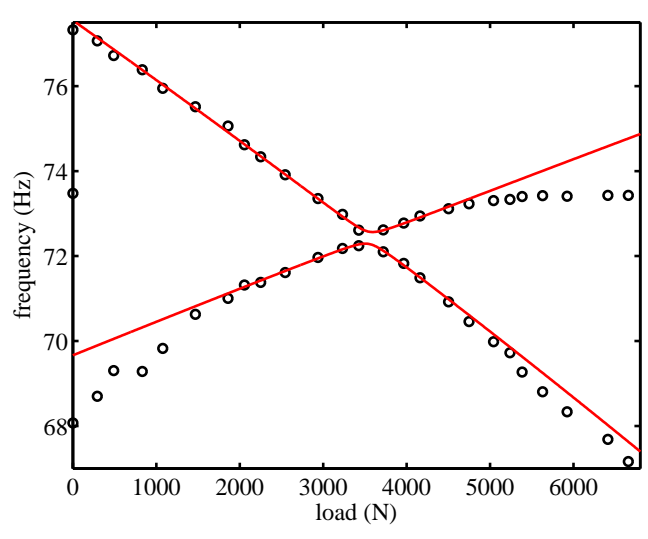

(a) Full range

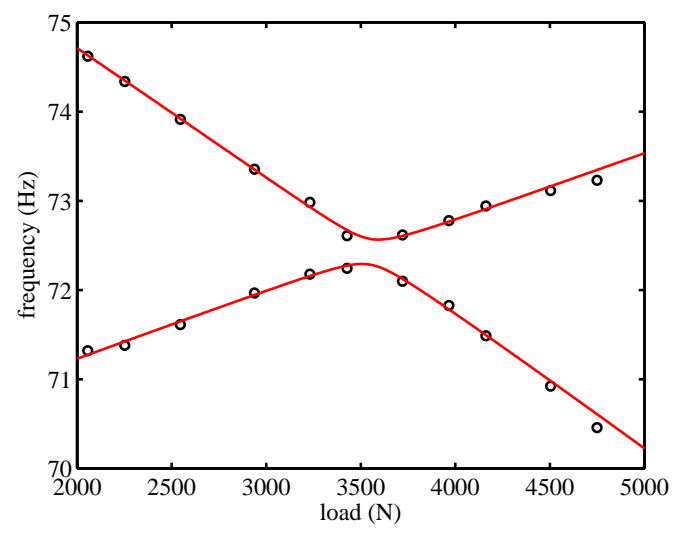

(b) Veering Region

Figure 5. The eigenvalue loci produced by the mathematical veering model ( - ). The upward inclined sections of the experimental frequency loci $(\circ)$ display significant nonlinear behaviour outside of the veering region. 
two rows of the matrix are used in any given update.

The sensitivity matrix for the first iteration is

$$
\mathbf{S}=\left[\begin{array}{cccc}
-473.7 & 473.7 & -420.9 & 420.9 \\
358.0 & 358.0 & 260.5 & 260.5 \\
2061 & 2061 & 1670 & 1670
\end{array}\right]
$$

From this it is clear that the vector rotation rate is the only parameter differentiating the symmetric parameters from one another. In addition it is seen that the two sets of symmetric parameters have similar sensitivities, confirming that the 4-parameter problem is still ill-conditioned. Accordingly, only the first two parameters will be considered in this example, corresponding to the weld stiffnesses at each end of the non-tensioned member (that is, the member which does not contain the tensioning mechanism).

The parameters are first updated using the vector rotation rate in conjunction with the veering parameter datum. Fig. 6(a-c) shows the parameter values and the updating variables' convergence history. As expected for equal numbers of parameters and variables, the variables converge exactly. The updated parameter values suggest that the weld stiffness is greater in the top left corner than the bottom right. Visual examination of the two welds in fig. 7 does not provide a rigorous confirmation of this result, although the weld bead does appear thicker in the top left corner and the base metal errosion greater in the lower right corner.

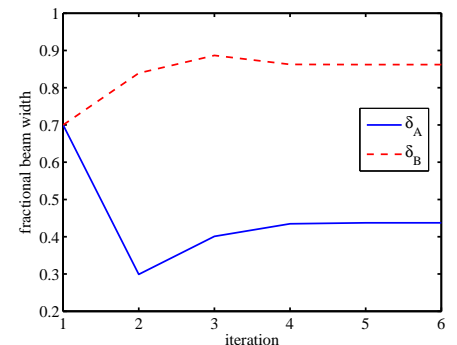

(a) Parameter convergence

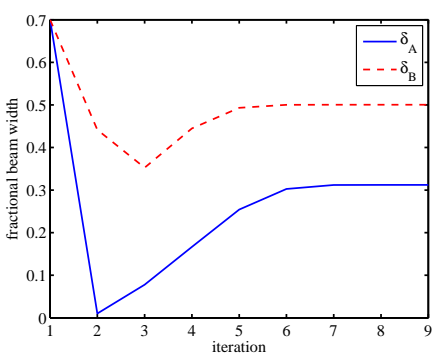

(d) Parameter convergence

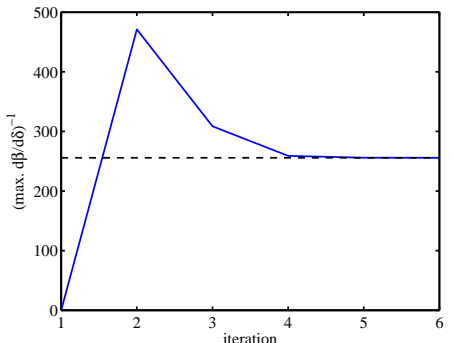

(b) Vector rotation rate convergence

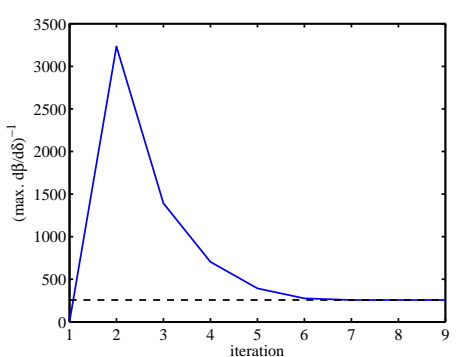

(e) Vector rotation rate convergence

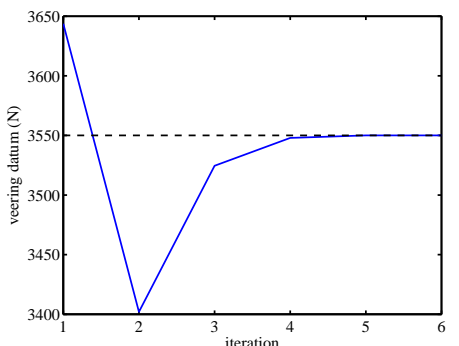

(c) Veering parameter datum convergence

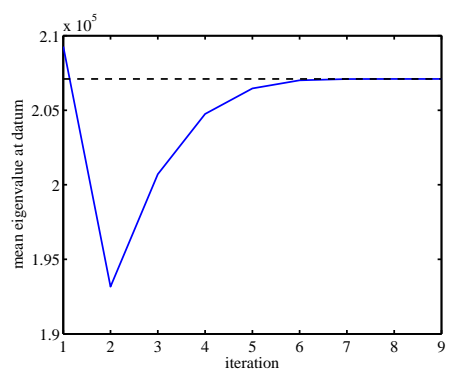

(f) Datum mean eigenvalue convergence

Figure 6. Parameter values and convergence history for the welded frame, updated using the eigenvector rotation rate and the veering parameter datum [(a)-(c)] or the mean eigenvalue at the datum [(d)-(f)]. Dotted lines indicate experimentally obtained values.

The updated FE model is then loaded incrementally to produce the eigenvalue loci in fig. 8(a). These are seen to reproduce the experimental trends well, although the eigenvalues are higher than those obtained in the expermental data.

Performing the update with respect to the mean eigenvalue at veering instead of the veering parameter datum produces similar results, seen in fig. 6(d-f), with the top left corner stiffer than the bottom right. Both weld stiffness parameters are seen to be lower to compensate for the high eigenvalues obtained in the first example, producing the eigenvalue loci in fig. 8(b). The compromise is that the veering parameter datum no longer matches that of the experiment. It is expected that a more extensive future study will incorporate enough experimental data to identify a unique solution matching all of the veering characteristics: an expectation that is justified by the successful separation of two symmetric parameters in the current results. 


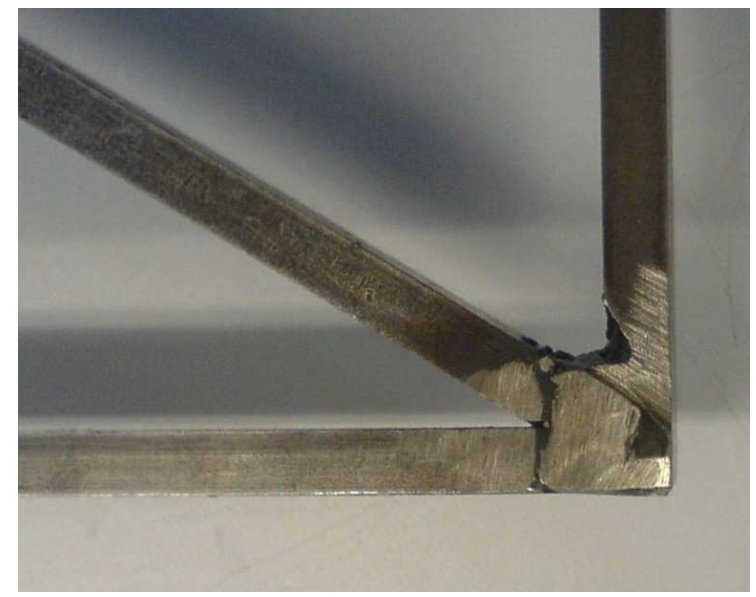

(a) Bottom right (A).

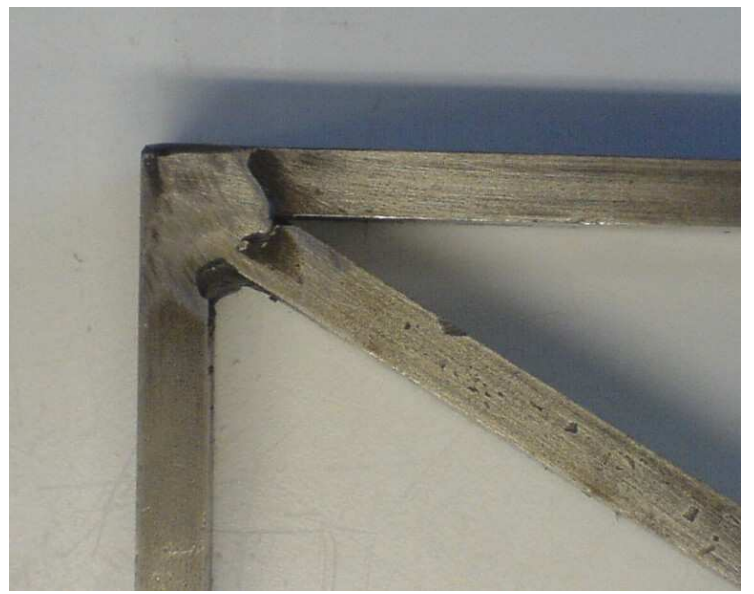

(b) Top left (B).

Figure 7. Welded joints in the corners; these weld stiffnesses are used as parameters in the model update.

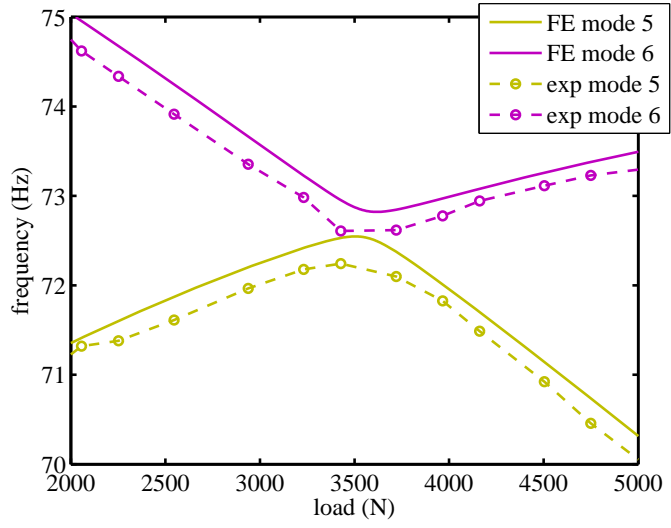

(a) veering parameter datum

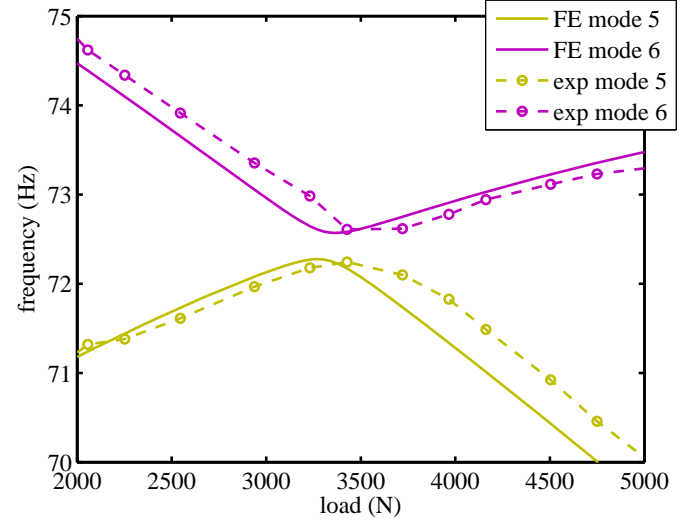

(b) veering parameter datum

Figure 8. The eigenvalues of the updated FE model compared to those of the experimental data. The eigenvector rotation rate was used in conjunction with the veering parameter datum or the mean eigenvalue at veering to produce these results. 


\section{Conclusions}

This paper has demonstrated the use of FE model updating to successfully reproduce characteristics of veering modes in dynamic systems. The examples presented are based upon very limited experimental data and consequently are incapable of updating all of the necessary parameters to reconcile the results exactly. It is seen, however, that the principles are sound and will produce excellent results when used in conjunction with a full experimental data set.

The updating scheme takes advantage of some quantities derived herein, specifically characterising the veering modes. In particular, the maximum rate of eigenvector rotation within a fixed subspace is found to be the most representative quantity for describing the severity of the veering. This quantity has the added advantage of being able to differentiate between variations in symmetric parameters; something that is often difficult to accomplish without careful mode shape measurements. This advantage has been demonstrated clearly in the results presented.

The computational derivation of the quantities is achieved efficiently using a single eigensolution, without the need to iterate solutions over the veering region. Similarly, robust techniques are described for determining the quantities from a range of experimental tests over the veering range. The theory has only been applied to stiffness variations but analogous derivations exist for mass variations. It is thought that the methods will provide efficient means for analysing a wide range of localisation and veering problems.

\section{Acknowledgments}

Thanks are due to the EPSRC and AgustaWestland for supporting this research through a research grant and CASE award.

\section{References}

${ }^{1}$ Hodges, C. H. and Woodhouse, J., "Vibration Isolation from Irregularity in a Nearly Periodic Structure: Theory and Measurements," Journal of the Acoustical Society of America, Vol. 74, No. 3, 1983, pp. 894-905.

${ }^{2}$ du Bois, J. L., Adhikari, S., and Lieven, N. A. J., "Eigenvalue Curve Veering in Stressed Structures: An Experimental Study," Journal of Sound and Vibration, 2009, published online, doi:10.1016/j.jsv.2008.12.014.

${ }^{3}$ Bendiksen, O., "Localization phenomena in structural dynamics," Chaos, Solitons and Fractals, Vol. 11, No. 10, 2000, pp. $1621-1660$.

${ }^{4}$ Perkins, N. C. and Mote, Jr., C. D., "Comments on Curve Veering in Eigenvalue Problems," Journal of Sound and Vibration, Vol. 106, No. 3, 1986, pp. 451-463.

${ }^{5}$ Ewins, D., "The effects of detuning upon the forced vibrations of bladed disks," Journal of Sound and Vibration, Vol. 9, No. 1, 1969, pp. 65-65.

${ }^{6}$ Afolabi, D. and Mehmed, O., "On Curve Veering and Flutter of Rotating Blades," Journal of Engineering for Gas Turbines and Power, Vol. 116, 1994, pp. 702-708.

${ }^{7}$ Balmès, E., "High Modal Density, Curve Veering, Localization: A Different Perspective on the Structural Response," Journal of Sound and Vibration, Vol. 161, No. 2, 1993, pp. 358-363.

${ }^{8}$ du Bois, J. L., Lieven, N. A. J., and Adhikari, S., "Localisation and Curve Veering: A Different Perspective on Modal Interactions," Proceedings of the 27th International Modal Analysis Conference, 2009.

${ }^{9}$ Friswell, M. I. and Mottershead, J. E., Finite Element Model Updating in Structural Dynamics, Kluwer Academic Publishers, 1995.

${ }^{10}$ Nelson, R. B., "Simplified Calculation of Eigenvector Derivatives," AIAA Journal, Vol. 14, No. 9, 1976, pp. 1201-1205.

${ }^{11}$ Fox, R. L. and Kapoor, M. P., "Rates of Change of Eigenvalues and Eigenvectors," AIAA Journal, Vol. 6, No. 12, 1968, pp. 2426-2429.

${ }^{12} \mathrm{du}$ Bois, J. L., Adhikari, S., and Lieven, N. A. J., "Experimental and Numerical Investigation of Mode Veering in a Stressed Structure," Proceedings of the IMAC, Vol. XXV, 2007, pp. 233. 